\title{
Caracterização do zumbido em idosos e de possíveis transtornos relacionados
}

\author{
Lidiane Maria de Brito Macedo Ferreira ${ }^{1}$, Alberto \\ Novaes Ramos Júnior ${ }^{2}$, Eveline Pereira Mendes ${ }^{3}$
}

\section{Resumo / Summary}

$\mathrm{O}$ envelhecimento populacional é uma realidade atual no Brasil, e com ele observa-se o aumento de doenças crônicodegenerativas. O zumbido surge como um sintoma muito prevalente e de alto impacto na qualidade de vida do paciente senil. Objetivo: Avaliar e qualificar o zumbido neste grupo. Material e Método: Questionário de pesquisa aplicado a 100 idosos em hospital terciário, aleatoriamente, com questões sobre características do zumbido; repercussão do zumbido na vida do paciente e antecedentes pessoais. Resultados: $61 \%$ dos participantes eram do sexo feminino, a média de idade foi de 69,53 anos. Em relação às características do zumbido: não-pulsátil $76 \%$, contínuo $54 \%$, bilateral $57 \%$, recente $62 \%$ e único 83\%; à repercussão: $32,5 \%$ referiram alteração no emocional, $31,8 \%$ no sono, $22,5 \%$ na concentração e $13,2 \%$ na vida social; $39 \%$ classificaram o zumbido em moderado, $35 \%$ em intenso e $26 \%$ em leve; às comorbidades: relação com sintomas otoneurológicos e hipertensão arterial; aos achados audiométricos: curvas descendentes, sensorioneurais e simétricas foram prevalentes. Conclusões: $\mathrm{O}$ zumbido interfere na vida do idoso; não há correlação entre o grau da perda auditiva e o grau de insatisfação do paciente com o zumbido; e a presbiacusia foi o achado mais comum encontrado nas audiometrias.

\section{Characterization of tinnitus in the elderly and its possible related disorders}

Palavras-chave: idoso, qualidade de vida, zumbido. Keywords: elderly, quality of life, tinnitus.

\begin{abstract}
$\mathrm{P}$ - opulation aging it is a current reality in Brazil and tinnitus appears as a very prevalent symptom, having a high impact on the quality of life of elderly patients. Aim: to evaluate and to characterize tinnitus in this group. Materials and Methods: A research questionnaire randomly given to 100 elderly patients in a tertiary hospital, asking about tinnitus characteristics, its impact on the life of the patient, and personal medical history. Results: $61 \%$ of the participants were female, average age average was 69.53 years. The results associated with tinnitus features were: no-pulsatile $76 \%$, continuous $54 \%$, bilateral $57 \%$, recent $62 \%$ and alone $83 \% ; 32,5 \%$ had emotional disorders, $31.8 \%$ had sleep disorders, $22.5 \%$ had difficulties concentration and $13.2 \%$ had social problems; $39 \%$ classified their tinnitus in moderate; 35\% had intense tinnitus and 26\% considered it a mild one: as to comorbidities: relation with neurotology symptoms and hypertension; as for associations with audiometry findings: descending, sensorineural and symmetrical curves prevailed. Conclusions: Tinnitus has a relevant impact on the lives of the elderly; there was no correlation between the level of hearing loss and the level of patient dissatisfaction caused by tinnitus; and presbycusis was the most common finding in the audiometric tests.
\end{abstract}

${ }^{1}$ Médica Otorrinolaringologista.

${ }^{2}$ Doutor e Médico infectologista - Departamento de Saúde Professor do Departamento de Saúde Comunitária da Faculdade de Medicina da Universidade Federal do Ceará

${ }^{3}$ Fonoaudióloga do setor de próteses auditivas do HGF.

Este artigo foi submetido no SGP (Sistema de Gestão de Publicações) da RBORL em 20 de outubro de 2007. cod. 4878 Artigo aceito em 27 de julho de 2008 . 


\section{INTRODUÇÃO}

O envelhecimento populacional é uma realidade em todo o mundo, mesmo em cenário de subdesenvolvimento. Idoso, de acordo com a Lei brasileira de número 8.842, de 04 de janeiro de 1994, é definido como a pessoa com idade superior a 60 anos. Já de acordo com o conceito da Organização Mundial da Saúde (OMS), idoso em cenários de países em desenvolvimento inclui pessoas com idade igual ou superior a 60 anos e a 65 anos para os países desenvolvidos ${ }^{1}$. Em escala mundial, a esperança de vida ao nascer foi estimada, para 2000, em 65 anos e, para 20452050, a Organização das Nações Unidas (ONU) projeta uma vida média de 74,3 anos ${ }^{2}$.

Dentre os processos mórbidos do idoso, podemos citar como de relevância clínica o zumbido, que apesar de não ser doença e sim um sintoma, pode refletir o funcionamento do organismo do indivíduo como um todo e é bastante prevalente nesta faixa etária. Afeta $15 \%$ da população em geral e 33\% dos idosos ${ }^{3}$. É uma desordem extremamente frequente, atingindo cerca de 40 milhões de pessoas nos EUA, das quais 10 milhões são gravemente afetadas por ele . $^{4}$ Pode ser causado por inúmeras afecções, sejam elas otológicas, metabólicas, neurológicas, ortopédicas, cardiovasculares, farmacológicas, odontológicas e psicológicas, as quais, por sua vez, podem estar presentes concomitantemente no mesmo indivíduo $0^{5,6}$.

Sataloff et al. ${ }^{4}$ relataram que aproximadamente $85 \%$ dos pacientes que procuram um otologista têm zumbido. Benevides $^{7}$ relatou que o zumbido é um sintoma que frequentemente acompanha a presbiacusia e costuma ser mais perturbador que a surdez. No Brasil não há dados absolutos de qual a prevalência de pacientes com zumbido, entretanto estima-se que esses dados assemelhem-se aos apresentados pelos países desenvolvidos. Isso se daria uma vez que o perfil epidemiológico do paciente idoso brasileiro revela-se parecido com o dos idosos dos países de primeiro mundo, no que diz respeito à presença de doenças crônico-degenerativas (que como discutido anteriormente, têm estreita relação com a gênese do zumbido).

Em 2004, em um estudo com 260 pacientes idosos na cidade de Fortaleza, com queixas auditivas, observouse que a queixa auditiva que prevaleceu foi o zumbido (58,08\%), confirmando sua alta prevalência dentre os pacientes idosos que procuram auxílio otorrinolaringológico8.

Frequentemente a presença do zumbido tornase um fator de grande repercussão negativa na vida do indivíduo, dificultando seu sono, sua concentração nas atividades diárias e profissionais, sua vida social e, muitas vezes, alterando sobremaneira seu equilíbrio emocional, acarretando estados de ansiedade e depressão,6,9-11.

Os aspectos psicológicos do zumbido já são muito bem conhecidos e discutidos por vários autores ${ }^{12-15}$. Segundo Sanchez ${ }^{16}$, a presença de perda auditiva aumenta o risco de o zumbido provocar interferência na concentração e no equilíbrio emocional ou que ela funcione como um cofator desta interferência.

O que motivou a realização desse estudo foi a observação clínica de que muitos idosos apresentam o zumbido como causa de inquietação e de graves problemas na sua saúde de uma forma global, com prejuízos nas suas funções diárias. Este estudo justifica-se, portanto, por: alta prevalência do zumbido na população idosa3; o fato de o zumbido ser tido como causa de diminuição da inteligibilidade da fala nesta população, dificultando seu relacionamento interpessoal; a presença de alterações do comportamento como irritabilidade e insônia, que potencializam quadros depressivos e o fato de o zumbido afetar as atividades de vida diária (AVDs) e as atividades instrumentais de vida diária (AIVDs).

Nossosobjetivos foram: traçar um perfil epidemiológico dos idosos portadores de zumbido atendidos em hospital terciário de Fortaleza - CE; caracterizar o zumbido nestes idosos; correlacionar a existência de comorbidades em idosos portadores de zumbido; identificar alterações audiométricas em idosos portadores de zumbido e quantificar a interferência do zumbido na vida do idoso.

\section{MATERIAL E MÉTODO}

Estudo do tipo transversal, descritivo, de natureza quantitativa.

Foi realizado no ambulatório de otorrinolaringologia de um hospital terciário em Fortaleza - Ceará, com um serviço de referência em otorrinolaringologia.Os participantes da pesquisa foram pacientes idosos (acima de 60 anos) que procuraram atendimento médico no Hospital, independente da especialidade, portadores de zumbido. Estes idosos foram captados através de avisos dispostos nos corredores do hospital explicando a existência da pesquisa. Foram incluídos os 100 primeiros idosos que compareceram à avaliação ambulatorial de otorrinolaringologia, de ambos os sexos e que consentirem participar da pesquisa. Como critérios de exclusão foram definidos: pacientes portadores de zumbidos causados por problemas agudos, tais como otites agudas ou rolhas de cerúmen e pessoas com limitação à realização do exame audiométrico, tais como déficit cognitivo ou respostas inconsistentes.O estudo foi realizado entre os meses de junho a dezembro de 2006. Na medida em que foram se apresentando ao ambulatório de otorrinolaringologia, os idosos realizaram anamnese complementar e exame físico otorrinolaringológico básico, todos realizados pela pesquisadora principal, e foram agendados para a realização de avaliação audiométrica executada por um único fonoaudiólogo no setor, para padronização. A coleta de dados foi realizada por meio da aplicação de um questionário padrão, adaptado de Sanchez ${ }^{16}$, contendo perguntas que contemplam: identificação com idade e sexo, características 
do zumbido, (tipo, frequência de percepção, localização, tempo de surgimento); a repercussão do zumbido na vida do pacientee antecedentes pessoais.

Após a realização dos exames, os idosos foram novamente avaliados pela pesquisadora principal e, dependendo dos achados audiológicos encontrados, foram encaminhados para tratamento específico dentro do próprio hospital.

A audiometria foi realizada com o audiômetro marca Interacoustics, modelo AD-28, em cabine acústica, por fonoaudióloga única. Para classificação da perda auditiva, utilizamos a classificação de Davis e Silverman ${ }^{17}$.

Em todos os casos nos quais observamos curvas audiométricas assimétricas, foram realizados exames de investigação tais como Potencial evocado de tronco encefálico e ressonância nuclear magnética de crânio, para afastar causas retrococleares.

A Análise de dadosdeu-se através da descrição de variáveis por meio de estatística descritiva e análise de contexto, com a composição de tabelas e gráficos. Utilizou-se o aplicativo Excel (Office ${ }^{\circledR}$ ) da Microsoft para a construção da planilha de dados, de tabelas e gráficos.As pessoas incluídas neste estudo tiveram a sua participação efetivada após o esclarecimento de todas as etapas do estudo, e após assinarem o termo de consentimento livre e esclarecido. A pesquisa foi analisada e aprovada pelo Comitê de Ética em Pesquisa do Hospitalem 01/06/2006, sob o registro número 010604/06.

\section{RESULTADOS}

Em relação à distribuição por gênero, dentre as 100 pessoas estudadas, observou-se uma frequência maior de mulheres idosas portadoras de zumbido $(61,0 \%)$, numa faixa etária maior entre 60 e 70 anos(Quadro 1, Gráficos $1,2$ e 3$)$.

Quadro 1. Estatística descritiva. Idade segundo sexo.

\begin{tabular}{|c|c|c|c|c|}
\hline & sexo & & Estat. & Erro Padrão \\
\hline \multirow[t]{24}{*}{ idade } & \multirow[t]{12}{*}{$\mathrm{F}$} & Média & 68,77 & ,839 \\
\hline & & \multirow{2}{*}{ Intervalo de Confiança de 95\% para a média } & Limite Inferior & 67,09 \\
\hline & & & Limite Superior & 70,45 \\
\hline & & Mediana & 67,00 & \\
\hline & & Variância & 42,913 & \\
\hline & & Desvio Padrão & 6,551 & \\
\hline & & Mínimo & 60 & \\
\hline & & Máximo & 88 & \\
\hline & & Variação & 28 & \\
\hline & & Var Interquartil & 9 & \\
\hline & & Skewness & ,711 & ,306 \\
\hline & & Kurtosis &, 141 & ,604 \\
\hline & \multirow[t]{12}{*}{ M } & Média & 70,72 & 1,315 \\
\hline & & Intervalo de Confiança de 95\% para a média & Limite Inferior & 68,06 \\
\hline & & & Limite Superior & 73,38 \\
\hline & & Mediana & 69,00 & \\
\hline & & Variância & 67,418 & \\
\hline & & Desvio Padrão & 8,211 & \\
\hline & & Mínimo & 60 & \\
\hline & & Máximo & 90 & \\
\hline & & Variação & 30 & \\
\hline & & Var Interquartil & 10 & \\
\hline & & Skewness & ,912 & ,378 \\
\hline & & Kurtosis & ,470 & ,741 \\
\hline
\end{tabular}




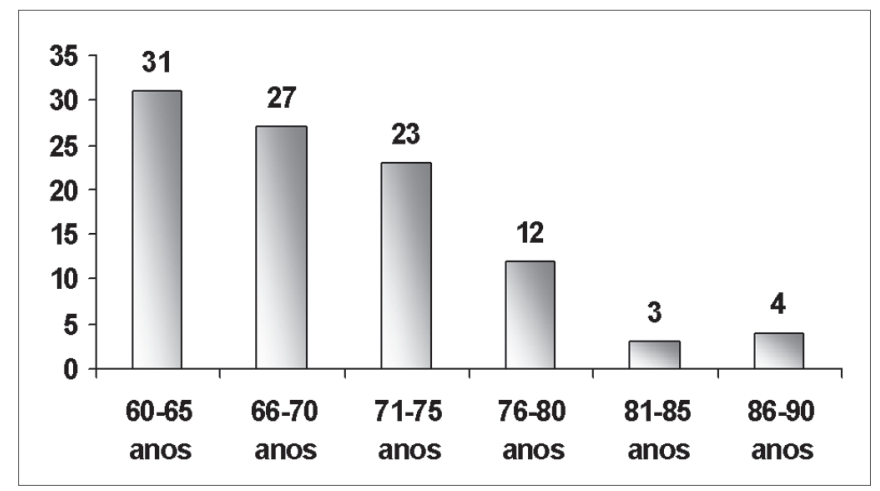

Gráfico 1. Distribuição do percentual dos pacientes $(n=100)$ com zumbido quanto à idade

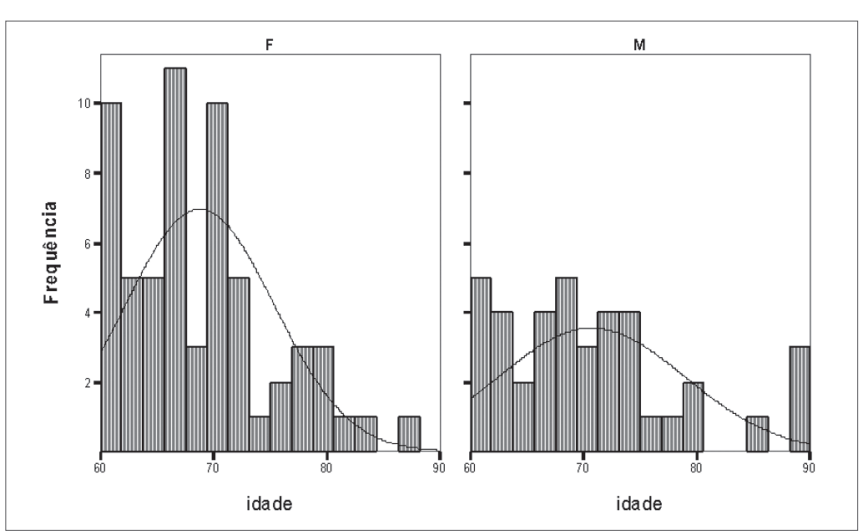

Gráfico 2. Distribuição etária dos pacientes $(n=100)$ com zumbido segundo sexo

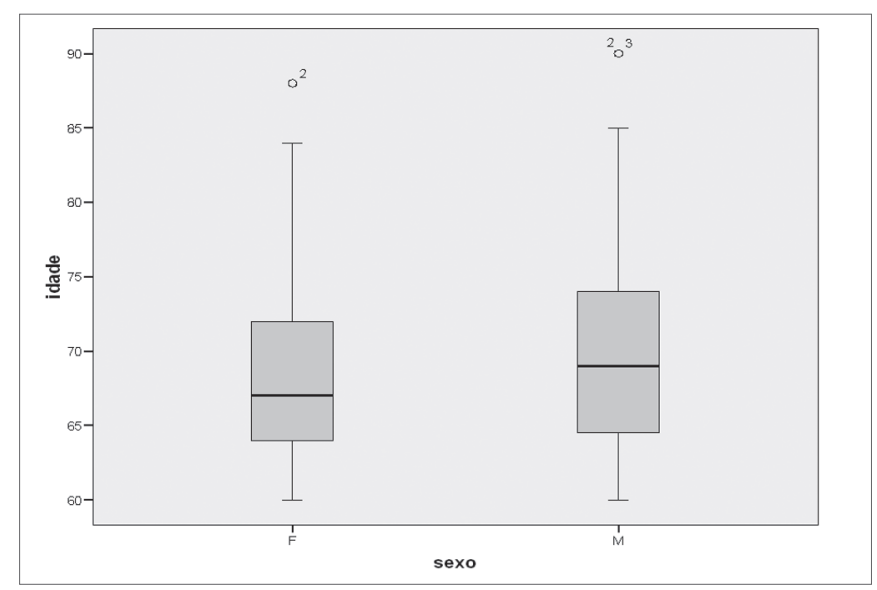

Gráfico 3. Análise da distribuição etária dos pacientes $(n=100)$ com zumbido segundo sexo

O zumbido foi caracterizado pelos pacientes principalmente sobre as formas não-pulsátil, contínuo, bilateral, recente e único. (Tabela 1 )

O incômodo do zumbido foi medido segundo informações sobre interferência em situações e momentos do dia-a-dia, segundo observado na Tabela 2 e no Gráfico 4.
Tabela 1. Características do zumbido presentes no grupo de idosos $(n=100)$.

\begin{tabular}{lc}
\hline \multicolumn{1}{c}{ Característica do Zumbido } & $\%$ \\
\hline Quanto ao Tipo & 24,0 \\
- Pulsátil & 76,0 \\
- Não-pulsátil & \\
Quanto à Frequência de Percepção & 46,0 \\
-Intermitente & 54,0 \\
-Contínuo & \\
Quanto à Localização & 43,0 \\
-Unilateral & 57,0 \\
-Bilateral & \\
Quanto ao Tempo & 62,0 \\
-Recente & 17,0 \\
-Médio tempo & 21,0 \\
-Longo tempo & \\
Quanto ao Tipo & 83,0 \\
-Único & 17,0 \\
-Múltiplo &
\end{tabular}

Tabela 2. Frequência da distribuição do zumbido quanto à sua repercussão na vida dos pacientes idosos $(n=100)$.

\begin{tabular}{llc}
\hline \multicolumn{1}{c}{ REPERCUSSÃO } & $\mathrm{N}$ & \% (ao total de interferência) \\
\hline Interfere no sono & 41 & 31,8 \\
Interfere na concentração & 29 & 22,5 \\
Interfere no emocional & 42 & 32,5 \\
Interfere na vida social & 17 & 13,2 \\
\hline
\end{tabular}

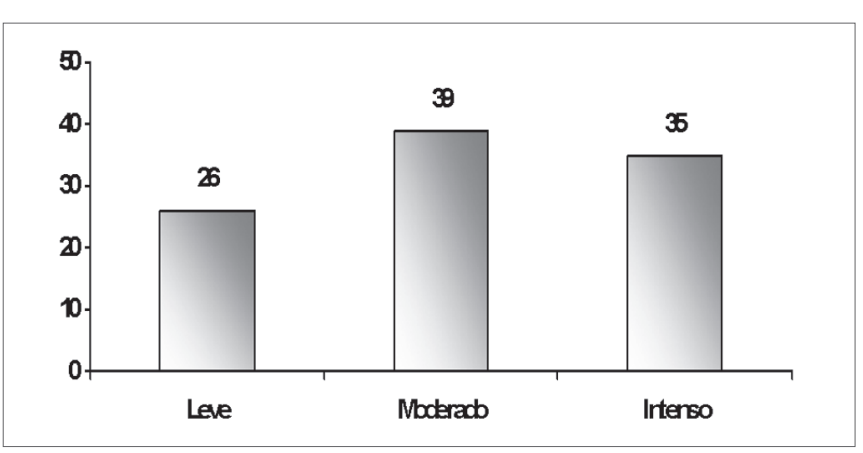

Gráfico 4. Distribuição percentual da avaliação de incômodo do zumbido pela escala Análogo-visual dos pacientes idosos $(n=100)$

Vários fatores, como já vistos, estão relacionados na gênese do zumbido, e no presente estudo foram encontrados fatores importantes, especialmente a hipertensão arterial e o uso de medicamentos (Tabela 3).

A perda auditiva é um sintoma frequentemente presente em associação ao zumbido, e suas características são identificadas nos Gráficos 5, 6 e 7, e na Tabela 4. 
Tabela 3. Fatores de risco relacionados à presença do zumbido em pacientes idosos $(n=100)$.

\begin{tabular}{lc}
\hline \multicolumn{1}{c}{ FATOR DE RISCO } & $\%$ \\
\hline Hipoacusia & 74,00 \\
Uso de medicamento & 74,00 \\
Vertigem & 52,00 \\
HAS & 49,00 \\
Dieta com estimulantes & 36,00 \\
Hipersensibilidade auditiva & 30,00 \\
Dislipidemia & 27,00 \\
DM & 12,00 \\
DVP & 12,00 \\
Tabagismo & 12,00 \\
Etilismo & 5,00 \\
\hline
\end{tabular}

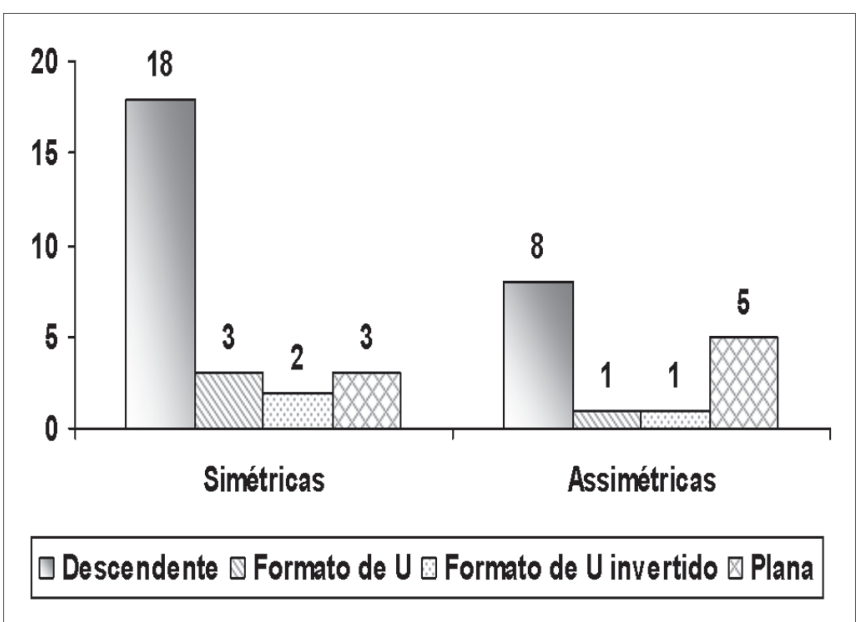

Gráfico 5. Distribuição percentual da avaliação de incômodo do zumbido pela escala Análogo-visual dos pacientes idosos $(n=100)$

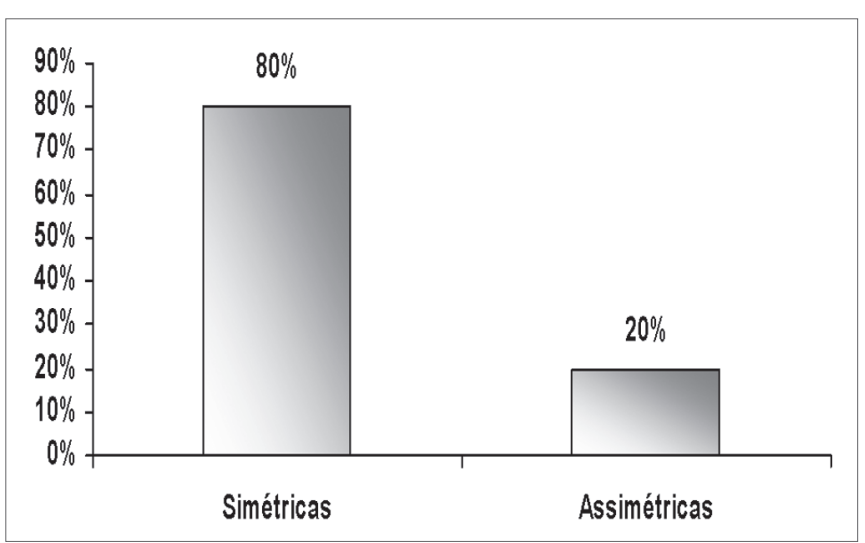

Gráfico 6. Distribuição das curvas audiométricas quanto à simetria interaural em pacientes idosos

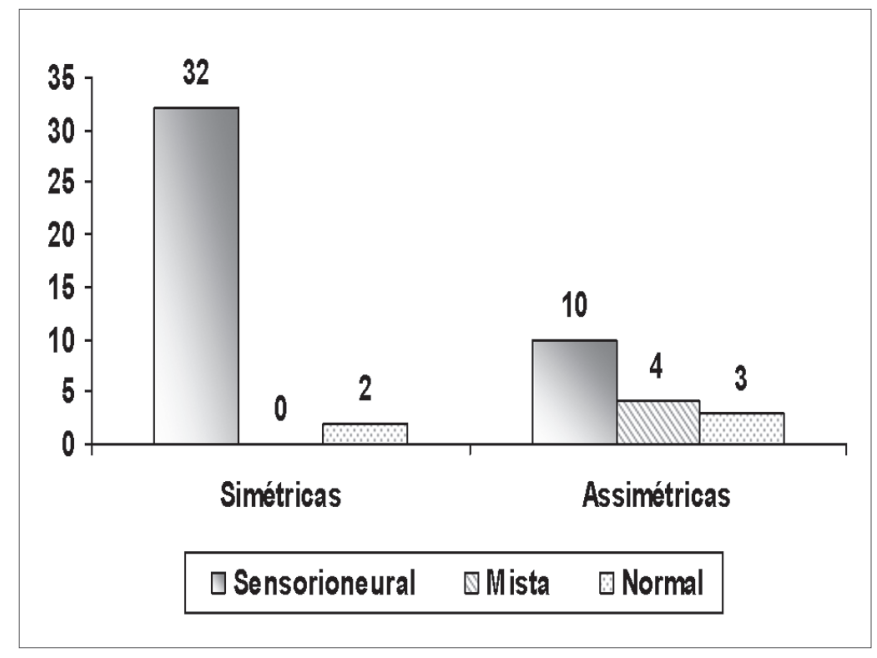

Gráfico 7. Distribuição das curvas audiométricas quanto à natureza da perda auditiva em pacientes idosos

Tabela 4. Percentual da relação entre interferência do zumbido e grau da perda auditiva em pacientes idosos $(n=100)$.

\begin{tabular}{cccccc}
\hline Zumbido & $\begin{array}{c}\text { Perda } \\
\text { leve }\end{array}$ & $\begin{array}{c}\text { Perda mode- } \\
\text { rada e mo- } \\
\text { deradamente } \\
\text { severa }\end{array}$ & $\begin{array}{c}\text { Perda } \\
\text { severa } \\
\text { profunda }\end{array}$ & $\begin{array}{c}\text { Sem } \\
\text { perda }\end{array}$ & Total \\
\hline Leve & 33,4 & 40,0 & 20,0 & 6,7 & 100,00 \\
Moderado & 23,1 & 50,0 & 23,1 & 3,8 & 100,00 \\
Intenso & 48,0 & 40,0 & 8,0 & 4,0 & 100,00 \\
\hline \multicolumn{5}{c}{ DISCUSSÃO } \\
\hline
\end{tabular}

Como a casuística envolveu pacientes que buscaram atendimento em qualquer clínica do hospital, essa tendência provavelmente refletiu o fato de que as mulheres frequentemente procuram mais atendimento médico que os homens.

Em relação à idade, a idade variou de 60 a 90 anos (variância de 30), com uma média de idade de 69,53 anos e desvio padrão de 7,26, compatível com a média encontrada em outros estudos que foi de 65,5 anos18. A mediana de idade foi de 69 anos, com moda de 71 anos. Em estudo realizado também em Fortaleza, em $2004^{8}$, o grupo etário mais frequente foi entre 65 e 70 anos, também confirmando os resultados (Gráficos 1, 2e 3).

As principais características atribuídas ao zumbido foram: caráter não-pulsátil, contínuo, bilateral, recente e único (Tabela 1). Person ${ }^{19}$ encontrou $36,0 \%$ para zumbido intermitente e 63,3\% para zumbido contínuo na sua casuística, confirmando nossos dados de que há um percentual maior de zumbido sob a forma contínua.

Quando perguntados a respeito da repercussão do zumbido em sua vida diária, a maioria dos pacientes referiu interferência no sono e no emocional (Tabela 2). Este 
dado reflete-se na nota de incômodo dada pelo paciente, que classifica o zumbido como incômodo acentuado em boa parte dos casos (Gráfico 4).

Oincômodo sentido pelo paciente é um sintoma bastante subjetivo e, muitas vezes, depende de fatores externos e psicológicos, além dos sentimentos negativos que ele atribui àquele zumbido. No nosso estudo, avaliamos o grau desse incômodo através de uma escala de zero a dez, ou por escala análogo-visual com faces, de triste a feliz, pontuando a insatisfação com o zumbido. Encontramos $39,0 \%$ dos pacientes com queixa de zumbido moderado, número aproximado ao encontrado na literatura $\left(49,0 \%{ }^{18} \mathrm{e}\right.$ $31,8 \%{ }^{19}$ ). Trinta e cinco relataram zumbido grave e $26,0 \%$, zumbido leve.

Sabe-se que comorbidades podem levar ao surgimento ou precipitarem um zumbido insipiente, e na nossa amostra observamos um alto percentual de idosos utilizando medicamentos (o que implica na polifarmácia conhecida entre os idosos) e hipertensos, além dos sintomas otológicos associados (Tabela 3). Na realidade, pouco se tem de concreto, mas é sabido pela prática clínica que alterações nos níveis pressóricos, glicêmicos, de zincemia ${ }^{19}$ e até mesmo de compostos da dieta (tais como xantinas) interferem na frequência do zumbido.

A hipertensão arterial como fator implicado na gênese do zumbido é ainda controversa. Brohen ${ }^{20}$, em 1996, analisando grupo de pacientes hipertensos, encontrou o zumbido como sintoma em 36,0\%; já Baraldi ${ }^{21}$, em 2004, correlacionou o zumbido com perda auditiva, e encontrou que $34,2 \%$ destes pacientes eram hipertensos. No nosso grupo, $49 \%$ dos idosos com zumbido eram hipertensos. Nossa pesquisa revelou alta correlação entre zumbido e hipertensão arterial, sendo que não houve significância entre o diabetes, a dislipidemia, a doença vascular periférica e a dieta com estimulantes. O uso de medicamentos para estas patologias foi encontrado em $74,0 \%$ dos pacientes, sendo que o grupo mais citado foi o de anti-hipertensivos. Antivertiginosos e medicamentos de ação central, tais como antidepressivos e benzodiazepínicos também foram citados.

O tabagismo e o etilismo não mostraram força na correlação com o zumbido, visto que representaram, respectivamente, $12,0 \%$ e $5,0 \%$ dos casos.

Já os sintomas otoneurológicos, esses são muito bem estudados e é visível sua associação com o zumbido. O zumbido, juntamente com a hipoacusia e a vertigem formam a tríade clássica da otoneurologia. Person ${ }^{19}$, em um estudo relacionando os sintomas otoneurológicos com a hipozincemia, detectou uma associação de $74 \%$ entre hipoacusia e zumbido, e de $52 \%$ entre vertigem e zumbido. Nossos dados revelaram associações de 59,1\% e $41 \%$, respectivamente, dados bem próximos à presença do zumbido.

A audiometria é um importante exame de scree- ning audiológico e otoneurológico, e na nossa casuística observamos grande maioria de curvas simétricas, sensorioneurais e descendentes, configuração típica do paciente idoso com presbiacusia (Gráficos 5, 6 e7). A simetria foi observada em 80,0\% dos casos, compatível com os dados de Amaral ${ }^{8}$, também de Fortaleza, que encontrou $73,8 \%$.

O incômodo gerado pelo zumbido é multiplicado à deficiência auditiva própria da idade (presbiacusia), levando ao isolamento social muitas vezes por dificuldade de comunicação. Os déficits de atenção, concentração e alterações no sono, já vigentes nesta idade, são aumentados na presença do zumbido, dificultando a realização das atividades instrumentais de vida diária e até mesmo gerando riscos de quedas.

A hipoacusia (como queixa do paciente, mas não comprovada audiologicamente) teve uma elevada associação nos nossos dados, correspondendo a 74,0\%. Já a vertigem mostrou-se presente em 52,0\%, e a hipersensibilidade auditiva em 30,0\%. O tipo específico desta hipoacusia está diretamente relacionado aos sintomas apresentados pelo paciente, visto que na sua maioria, a presbiacusia leva a um desequilíbrio na homeostase labirintococlear, podendo levar a vertigens e zumbidos associados, geralmente bilaterais por acompanhar a hipoacusia. Já nos casos de assimetria auditiva ou do zumbido, o alerta para patologias retrococleares ou neurais fica mais evidente, devendo ser pesquisados outros sintomas sistêmicos associados. Nossa casuística confirma a imensa maioria de perdas auditivas sensorioneurais descendentes bilaterais, características da presbiacusia, como principal fator associado ao zumbido em idosos. Isso se dá pela perda neural nas células ciliadas cocleares com o tempo. Todos os casos de perdas assimétricas foram investigados com exames de imagem cerebrais, sendo que nenhuma patologia retrococlear foi identificada.

A perda auditiva unilateral ou bilateral assimétrica foi encontrada em $26,9 \%$ dos casos; a perda auditiva bilateral simétrica em 65,4\%; e 7,7\% dos casos não possuíam perda auditiva. Não encontramos correlação entre o grau de perda auditiva medido pela audiometria e o nível de incômodo do zumbido relatado pelo paciente, o que comprova que o incômodo está relacionado à forma como o paciente encara seu zumbido, e não a alguma medida física ou anatômica (Tabela 4).

\section{CONCLUSÕES}

No presente estudo, observou-se que o zumbido é um fator de intensa insatisfação no paciente idoso, por prejudicar suas atividades diárias e proporcionaralterações do sono e do emocional. Isto deveria ser encarado com importância em qualquer tratamento clínico, na adesão às politerapias farmacológicas que o idoso em geral faz uso, além da interferência com processos depressivos já instalados ou não, comuns em idosos. 
Pudemos, ainda, correlacionar a presença hipertensão arterial nos pacientes com zumbido e a coexistência frequente de outros sintomas otoneurológicos. A presbiacusia, como era de se esperar, revela-se como a principal patologia associada em nível de achados audiométricos nesta população. E, finalmente, que não há correlação entre o grau audiométrico da perda auditiva e o nível de incômodo sentido pelo paciente, de modo que o sistema límbico está muito mais envolvido na gênese e perpetuação do zumbido que o próprio sistema auditivo.

\section{REFERÊNCIAS BIBLIOGRÁFICAS}

1. Santana RLF, Pouchain GC, Bissi LF. A previdência social e o Censo 2000: perfil dos idosos. Informe de previdência Social. 2002; 14(9):1-19.

2. Fundação Instituto Brasileiro de Geografia e Estatística (IBGE). Diretoria de Pesquisas. Coordenação de População e Indicadores Sociais. Gerência de Estudos e Análises da Dinâmica Demográfica. (Oliveira JC, Albuquerque FRPC), 2005. A mortalidade no Brasil no período 1980-2004: desafios e oportunidades para os próximos anos. Disponível em: http://www.ibge.gov.br/home/estatistica/populacao/ tabuadevida/2004/metodologica.pdf. Acessado em 03 de Março de 2006.

3. Seidmann MD, Jacobson GP. Update on tinnitus. Otolaryngol Clin North Am. 1996;29:455-65.

4. Sataloff J, Sataloff RT, Lueneburg W. Tinnitus and vertigo in healthy senior citizens without a history of noise exposure. Am J Otol. 1987;8(2):87-9.

5. Sanchez TG. Zumbido: estudo da relação entre limiar tonal e eletrofisiológico e das respostas elétricas do tronco cerebral. São Paulo, 1997 (Tese de Doutorado, Faculdade de Medicina da Universidade de São Paulo).

6. Sanchez TG, Zonato AY, Bittar RSM, Bento RF. Controvérsias sobre a Fisiopatologia do Zumbido. Arq Fund Otorrinolaringol. 1997b;1(1):2-8.
7. Benevides W. Otorrinolaringologista. Rio de Janeiro: Cultura Médica. 1997.

8. Amaral LCG, Sena APRC. Perfil audiológico dos pacientes da terceira idade atendidos no Núcleo de Atenção Médica Integrada da Universidade de Fortaleza. Fono Atual. 2004;7(27):58-64.

9. Dobie RA. Depression and tinnitus. Otolaryngol Clin North Am. 2003;36(2):383-8.

10. Sanchez TG. Zumbido: Análise crítica de uma experiência de pesquisa. São Paulo; 2003. (Tese de Livre-Docência, Faculdade de Medicina da Universidade de São Paulo).

11. Fowler EP. Head noises in normal and in normal and disordered ears: significance, measurement, differentiation and treatment. Arch Otolaryngol. 1944;39:498.

12. Mckee GJ, Stephens SD. An investigation of normally hearing subjects with tinnitus. Audiology. 1992;31(6):313-7.

13. Sanchez TG, Bento RF, Miniti A, Câmara J. Zumbido: características e epidemiologia. Experiência do Hospital das Clínicas da Faculdade de Medicina da Universidade de São Paulo. Rev Bras Otorrinolaringol. 1997;63(3):229-35.

14. Vallianatou NG, Christodoulou P, Nestoros JN, Helidonis E. Audiologic and Psychological profile of Greek patients with tinnitus - Preliminary findings. Am J Otol. 2001;22(1):33-7.

15. Jastreboff PJ. Phantom auditory perception (Tinnitus): mechanisms of generation and perception. Neurosci Res. 1990;8:221-54.

16. Sanchez TG, Medeiros IRT, Levy CPD, Ramalho JR, Bento RF. Zumbido em pacientes com audiometria normal: caracterização clínica e repercussões. Rev Bras Otorrinolaringol. 2005;71(4):427-31.

17. Davis H, Silverman SR. Hearing and deafness. 4th ed. New York, Holt, Rinehart and Winston, 1970.

18. Moura LOS, Iório MCM, Azevedo MF.A eficácia da adaptação de prótese auditiva na redução ou eliminação do zumbido. Rev Bras Otorrinolaringol. 2004;70(5):624-31.

19. Person OC, Nardi JC, Feres MCLC. A relação entre hipozincemia e zumbido. Rev Bras Otorrinolaringol. 2004;70(3):361-7.

20. Brohen VMA, Caovilla HH, Ganança MM. Sintomas e achados audiológicos e vestibulares em indivíduos com hipertensão arterial. Acta AWHO. 1996;15(1):4-10.

21. Baraldi GS, Almeida LC, Borges ACLC.Perda auditiva e hipertensão: achados em um grupo de idosos Rev Bras Otorrinolaringol. 2004;70(5):640-4. 